\title{
, \\ Infrasonic, Acoustic and Seismic Waves Produced by the Axion Quark Nuggets
}

\author{
Dmitry Budker ${ }^{1,2}{ }^{(D}$, Victor V. Flambaum ${ }^{3}\left[\right.$ and Ariel Zhitnitsky ${ }^{4, *}$ \\ 1 Johannes Gutenberg-Universität Mainz-Helmholtz-Institut, GSI Helmholtzzentrum für \\ Schwerionenforschung, 55128 Mainz, Germany; budker@uni-mainz.de \\ 2 Department of Physics, University of California, Berkeley, CA 94720-7300, USA \\ 3 School of Physics, University of New South Wales, Sydney 2052, Australia; v.flambaum@unsw.edu.au \\ 4 Department of Physics and Astronomy, University of British Columbia, Vancouver, BC 170-6371, Canada \\ * Correspondence: arz@phas.ubc.ca
}

\begin{abstract}
We advocate the idea that Axion Quark Nuggets (AQN) hitting the Earth can be detected by analysing the infrasound, acoustic, and seismic waves which always accompany their passage in the atmosphere and underground. Our estimates for the infrasonic frequency $v \simeq 5 \mathrm{~Hz}$ and overpressure $\delta p \sim 0.3$ Pa for relatively large size dark matter (DM) nuggets suggest that sensitivity of presently available instruments is already sufficient to detect very intense (but very rare) events today with existing technology. A study of much more frequent but less intense events requires a new type of instrument. We propose a detection strategy for a systematic study to search for such relatively weak and frequent events by using distributed acoustic sensing and briefly mention other possible detection methods.
\end{abstract}

Keywords: dark matter; axion; quark nuggets

check for updates

Citation: Budker, D.; Flambaum, V.V.; Zhitnitsky, A. Infrasonic, Acoustic and Seismic Waves Produced by the Axion Quark Nuggets. Symmetry 2022, 14, 459. https://doi.org/ $10.3390 /$ sym 14030459

Academic Editor: Alberto Ruiz Jimeno

Received: 11 January 2022

Accepted: 23 February 2022

Published: 24 February 2022

Publisher's Note: MDPI stays neutral with regard to jurisdictional claims in published maps and institutional affiliations.

Copyright: () 2022 by the authors Licensee MDPI, Basel, Switzerland. This article is an open access article distributed under the terms and conditions of the Creative Commons Attribution (CC BY) license (https:// creativecommons.org/licenses/by/ $4.0 /)$.

\section{Introduction and Motivation}

The main goal of the present work is to present a new method to detect axion quark nuggets (AQN) propagating in the Earth's atmosphere and underground.

The AQN dark matter model [1] was introduced decades ago to explain the observed similarity between the densities of dark and visible matter in the Universe, i.e.,

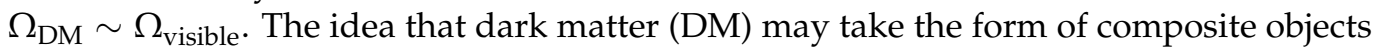
of standard-model quarks in a novel phase goes back to quark nuggets [2], strangelets [3], and nuclearities [4], see also the review [5] with references to the original results. In the models [2-5], the presence of strange quarks stabilizes the quark matter at sufficiently high densities, allowing strangelets formed in the early universe to remain stable over cosmological timescales. This type of DM is "cosmologically dark" not because of the weakness of the AQN interactions, but due to their small cross-section-to-mass ratio, which scales down many observable consequences of an otherwise strongly-interacting DM candidate. We review the basic ideas, predictions, and consequences of this model in Section 2 and mention the crucial ingredients here.

There are several additional elements in the AQN model in comparison with the older well-known and well-studied theoretical constructions [2-5]. First, there is an additional stabilization factor for the nuggets provided by the axion domain walls which are copiously produced during the quantum chromodynamic (QCD) transition, which help to alleviate a number of problems with the original nugget model [2-5]. Another feature of AQN which plays a crucial role in the present work is that nuggets can be made of matter as well as antimatter during the QCD transition. A direct consequence of this feature, given that the total baryon charge of the universe is zero, is that DM density, $\Omega_{\mathrm{DM}}$, and the

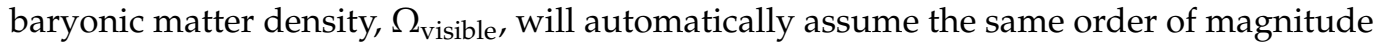
$\Omega_{\mathrm{DM}} \sim \Omega_{\text {visible }}$ without any fine tuning. 
One should emphasize that AQNs are absolutely stable configurations on cosmological scales. The antimatter which is hidden in the form of the dense nuggets is unavailable for annihilation unless the AQNs hit stars, planets, or interstellar media. The AQNs composed of antimatter are capable of releasing a significant amount of energy when they enter the Earth's atmosphere and annihilation processes occur between antimatter in the AQNs and atmospheric material.

How can this enormous amount of energy manifest itself? What would be the best means of detecting the corresponding effects caused by the AQN-annihilation processes? The problem is that the weakly interacting particles (such as axions and neutrinos) are hard to detect due to their weak interactions, while relatively strongly interacting photons and leptons have short mean free paths such that it is hard to recover the origin of these particles.

Indeed, the corresponding emission of photons in dilute environments such as the galactic center and at the locations of high-altitude Earth orbits can be studied and properly analyzed because the mean free path in such dilute environments is long. This should be contrasted with the case of dense environments where the annihilation events occur in the Earth's atmosphere when the energy is released in the form of the weakly coupled axions, neutrinos as well as $X$ and $\gamma$ rays. It is hard to observe axions and neutrinos due to their feeble interactions, though the corresponding computations have been carried out recently, see recent brief review [6] for references and details. At the same time, the $X$ and $\gamma$ rays emitted by AQNs are absorbed over short distances $\sim 10 \mathrm{~m}$ or so in the atmosphere, and therefore cannot be easily recovered for analysis due to the background radiation.

In this work, we propose that AQN-induced signals can be studied by analysing the acoustic waves which may propagate over large distances due to the large absorption lengths for such waves. Furthermore, we argue that the corresponding signal can be discriminated from background noise. Therefore, in this work we describe a new strategy for AQN detection based on their acoustic and seismic manifestations.

The presentation is organized as follows. In Section 2 we review the AQN model in the context of the present work, paying special attention to the size distribution, frequency of appearance, and the energy-emission pattern. In Section 3, we present our estimates for the AQNs propagating in the atmosphere and underground. The main lesson of this analysis is that the presently available instruments are capable of detecting signals produced by the large-size and intense AQNs which occur only once every 10 years or so. However, the presently available technical tools are not sufficiently sensitive to study typical and relatively small AQNs. Therefore, in Section 4, we present a proposal for a systematic study of acoustic and seismic events originating from AQNs with relatively small typical size which occur approximately once a day. We propose to employ distributed acoustic sensing which uses optical-fiber cables. In Appendix A as a side note we describe the observed mysterious event which occurred on July 31st 2008 and was properly recorded by the dedicated Elginfield Infrasound Array (ELFO) [7], near London, Ontario, Canada. The infrasound detection was not accompanied by any observations of meteors by an all-sky camera network. However, the signal was correlated with seismic signals in the area. It is tempting to identify the mysterious event recorded by ELFO with an intense (and rare) AQN-annihilation event, which has precisely the required features as described in Section 3.

\section{AQN Model: The Basics}

In this section, we review the basic ideas of the AQN model, its motivation, consequences, and (as of yet) indirect, rather than direct, supporting observations.

The original motivation for the model can be explained as follows. It is commonly assumed that the universe began in a symmetric state with zero global baryonic charge and later (through some baryon-number-violating process, nonequilibrium dynamics, and $\mathcal{C P}$-violation effects, realizing the three famous Sakharov criteria) evolved into a state with a net positive baryon number.

As an alternative to this scenario, we advocate a model in which "baryogenesis" is actually a charge-separation (rather than charge-generation) process in which the global 
baryon number of the universe remains zero at all times. In this model, the unobserved antibaryons consist of dark matter in the form of dense nuggets of antiquarks and gluons in the color superconducting (CS) phase. The result of this "charge-separation process" is two populations of AQN carrying positive and negative baryon number. In other words, the $A Q N$ may be formed of either matter or antimatter. However, due to the global $\mathcal{C P}$ violating processes associated with the so-called initial misalignment angle $\theta_{0}$ which was present during the early formation stage, the number of nuggets and antinuggets will be different. This difference is always an order-of-one effect irrespective of the parameters of the theory, the axion mass $m_{a}$ or the initial misalignment angle $\theta_{0}$. We refer to a short overview [6] on specific questions related to nugget formation, generation of the baryon asymmetry, and survival pattern of the nuggets during the evolution in the early universe. For the present studies, we take an agnostic viewpoint regarding the questions of formation of the $\mathrm{AQNs}$, and assume that such nuggets made of antimatter are present in our universe. This assumption is consistent with all available constraints as long as the average baryon charge of the nuggets is sufficiently large.

The key parameter which essentially determines all the intensities for the effects mentioned above is the average baryon charge $\langle B\rangle$ of the AQNs. There are a number of constraints on this parameter which are reviewed below. One should also mention that the AQN mass is related to its baryon charge by $M_{N} \simeq m_{p}|B|$, where we ignore small differences between the energy per baryon charge in CS and hadronic confined phases. The AQNs are macroscopically large objects with a typical size of $R \simeq 10^{-5} \mathrm{~cm}$ and roughly nuclear density of order $10^{40} \mathrm{~cm}^{-3}$ resulting in masses of roughly $10 \mathrm{~g}$.

An event where an AQN impinges on the Earth should be contrasted with conventional meteors. A conventional object with mass $10 \mathrm{~g}$ would have a typical size of order $1 \mathrm{~cm}$ occupying the volume which would be 15 orders of magnitude larger than the AQN volume. This is due to the fact that AQNs have nuclear density which is 15 orders of magnitude higher than the density of normal matter. One can view an AQN as a small neutron star (NS) with its nuclear density. The difference is that a NS is squeezed by gravity, while an AQN is squeezed by the axion-domain-wall pressure. The drastic density difference between AQNs and conventional small meteors leads to fundamentally different interaction patterns when they enter the Earth's atmosphere.

We now turn to the observational constraints on such dense objects. The strongest direct-detection limit is set by the IceCube Observatory, see Appendix A in [8]:

$$
\langle B\rangle>3 \times 10^{24} \quad \text { [direct (non)detection constraint] } .
$$

The basic idea of the estimate [8] is as follows. Using average flux density of AQNs computed below (5), one can obtain the total hits expected to IceCube in 10 years in form $\left\langle N_{\text {IceCube }}^{10 y r}\right\rangle$. Assuming a Poisson distribution, one can compute the probability of observing zero events within 10 years. The probability of seeing zero events in IceCube over 10 years leads to the estimate (1). In this estimate we obviously assume that such a large event as the AQN hit cannot be missed by the IceCube Observatory, i.e., we assumes $100 \%$ efficiency of the observation if the AQN hits the area. This assumption is similar to other studies such as magnetic monopole search.

The authors of [9] used the Apollo data to constrain the abundance of quark nuggets in the range of $10 \mathrm{~kg}$ to one ton. They argued that the contribution of such heavy nuggets must be at least an order of magnitude less than would saturate the dark matter in the solar neighbourhood [9]. Assuming that the AQNs do saturate dark matter, the constraint [9] can be reinterpreted as at least $90 \%$ of the AQNs having masses below $10 \mathrm{~kg}$. This constraint can be expressed in terms of the baryon charge:

$$
\left.B \lesssim 10^{28} \quad \text { [Apollo constraint }\right] .
$$


Therefore, indirect observational constraints (1) and (2) suggest that if the AQNs exist and saturate the dark matter density today, the dominant portion of them must reside in the window:

$$
3 \times 10^{24} \lesssim B \lesssim 10^{28} \text { [constraints from observations] }
$$

We emphasize that the AQN model with the limits (3) is consistent with all presently available cosmological, astrophysical, satellite, and ground-based constraints. This model is rigid and predictive since there is little flexibility or freedom to modify the fundamental parameters mentioned above. This comment applies to the model itself, not to the interactions with environment, which could be complex and often require introduction of unknown phenomenological parameters. In particular, the AQN flux (5) which plays a key role in the present studies cannot change by more than a factor of two, depending on the size distribution within the window (3).

It is important that the frequency of appearance of AQNs depends on the size distribution $f(B)$ defined as follows: Let $\mathrm{d} N / \mathrm{d} B$ be the number of AQNs which carry the baryon charge $[B, B+d B]$. The mean value of the baryon charge $\langle B\rangle$ is given by

$$
\langle B\rangle=\int_{B_{\min }}^{B_{\max }} \mathrm{d} B B f(B), \quad f(B) \propto B^{-\alpha},
$$

where $f(B)$ is a properly normalized distribution and $\alpha \simeq(2-2.5)$ is the power-law index. One should emphasize that the parametrization (4) was suggested in solar physics studies to fit the observed extreme UV radiation from the entire solar surface. We adopted this scaling in $[10,11]$, where it was proposed that the so-called nanoflares (conjectured by Parker many years ago to resolve the "Solar Corona Mystery") can be identified with AQN-annihilation events in the solar corona. The main motivation for this identification is that the observed intensity of the extreme UV emission from the solar corona matches the total energy released as a result of the AQN-annihilation events in the transition region assuming the conventional value for the dark matter density around the Sun, $\rho_{\mathrm{DM}} \simeq 0.3 \mathrm{GeVcm}^{-3}$. One should emphasize that this "numerical coincidence" is a highly nontrivial self-consistency check of the proposal $[10,11]$ connecting nanoflares with AQNs, since nanoflare properties are constrained by solar corona-heating models, while the intensity of the extreme UV due to the AQN annihilation events is mostly determined by the dark matter density. Furthermore, the required energy interval for the nanoflares must be in the range: $E_{\text {nano }} \simeq\left(10^{21}-10^{26}\right)$ erg. This allowed interval largely overlaps with the AQN baryonic charge window (3) if the identification between nanoflares and AQN annihilation events is made. In this case, $E_{\text {nano }} \simeq 2 m_{p} c^{2} B \simeq\left(3 \times 10^{-3} \mathrm{erg}\right) \times B$, see $[10,11]$ for details. One should note that the algebraic scaling (4) is a generic feature of the AQN-formation mechanism based on percolation theory [12], but $\alpha$ cannot be theoretically computed in strongly coupled QCD.

We now estimate the rate at which AQNs hit the Earth assuming the local dark matter density of $\rho_{\mathrm{DM}} \simeq 0.3 \mathrm{GeVcm}^{-3}$. Assuming the conventional halo model, one arrives at [8]:

$$
\begin{aligned}
\frac{\langle\dot{N}\rangle}{4 \pi R_{\oplus}^{2}} & \simeq \frac{4 \times 10^{-2}}{\mathrm{~km}^{2} \mathrm{yr}}\left(\frac{\rho_{\mathrm{DM}}}{0.3 \frac{\mathrm{GeV}}{\mathrm{cm}^{3}}}\right)\left(\frac{\left\langle v_{\mathrm{AQN}}\right\rangle}{220 \frac{\mathrm{km}}{\mathrm{s}}}\right)\left(\frac{10^{25}}{\langle B\rangle}\right) \\
\langle\dot{N}\rangle & \simeq 0.67 \mathrm{~s}^{-1}\left(\frac{10^{25}}{\langle B\rangle}\right) \simeq 2.1 \times 10^{7} \mathrm{yr}^{-1}\left(\frac{10^{25}}{\langle B\rangle}\right) .
\end{aligned}
$$

In deriving (5) it was assumed that $\langle\dot{N}\rangle \approx 4 \pi b^{2} n_{\mathrm{AQN}} v_{\mathrm{AQN}}$, where $b \approx 1.0013 R_{\oplus}$ is the impact parameter for Earth, and we averaged over incident angles of impacting AQNs, see Appendix C in [8] with details. We also assume that the AQNs represent the dominant portion of the DM. Numerical expression for (5) for the Earth and similar relation for the Sun were derived in [8] and in [11], respectively, using full-scale Monte Carlo simulations which account for all types of AQN trajectories with different $\mathrm{AQN}$ masses $M_{N} \simeq m_{p}|B|$, 
different incident angles, different initial velocities and size distributions. It was shown that none of these factors significantly affect our estimate. The result (5) suggests that AQNs hit the Earth's surface with a frequency on the order of once a day (i.e., hundreds per year) per $100 \times 100 \mathrm{~km}^{2}$. This rate is suppressed for large AQNs according to the distribution function (4).

It is instructive to compare the rate (5) with the number of meteoroids which enter the Earth's atmosphere. This rate is of the order of $10^{8} \mathrm{day}^{-1}$, see review [13]. It is more informative to represent this rate in terms of the total mass of the falling meteoroids, which is $10^{5}$ tons / year and is much greater than the total mass of order $5 \times 10^{2}$ tons / year associated with the dark matter AQN rate (5). The size distribution for meteoroids peaks at $2 \times 10^{-2} \mathrm{~cm}$ while the mass distribution peaks at around $10 \mu \mathrm{g}$, see [13]. This should be contrasted with a typical AQN size of $R \simeq 10^{-5} \mathrm{~cm}$ and mass of roughly $10 \mathrm{~g}$. The final topic we mention here is the spectral properties of the emission resulting from AQN propagating in matter. The most important feature of this spectrum which distinguishes it from meteor emission is that the AQN spectrum peaks in the 10 to $50 \mathrm{keV}$ range, while the optically visible bands at 1-10 eV are strongly suppressed, see Figure A4 in Appendix B. Another crucial difference with meteor emission is that the AQN spectrum is not thermal blackbody radiation as it originates from the annihilation events. It should be contrasted with the blackbody radiation of conventional meteors and meteorites entering the Earth's atmosphere with supersonic velocities and experiencing friction with the surrounding material resulting in heating of the meteoroids and surrounding material. We refer to Appendix B discussing the spectral features of the AQNs traversing the atmosphere, see in particular Figure A4.

These properties of the spectrum imply that the AQNs may avoid observation by conventional optical monitoring as AQNs are not accompanied by significant emission of the visible light and may not be routinely observed by all-sky cameras. Therefore, the observation of a signal by infrasound instruments and non-observation by the optical synchronized cameras (which must continuously monitor the sky recording conventional meteors) would eliminate spurious events and provide evidence of the AQNs entering the atmosphere.

We conclude this brief review section with Table 1 which summarizes the basic features of the AQNs. The parameter $\kappa$ in Table 1 is introduced to account for the fact that not all matter striking the nugget will annihilate and not all of the energy released by annihilation will be thermalized in the nuggets, see Appendix $B$ for the details. The ratio $\Delta B / B \ll 1$ in the Table implies that only a small portion of the (anti)baryon charge hidden in form of the AQNs get annihilated during big-bang nucleosynthesis (BBN), Cosmic Microwave Background (CMB), or post-recombination epochs, while the dominant portion of the baryon charge survives until the present time. The absolute stability of the AQNs in vacuum is a result of the energy per baryon charge in (anti)quark nuggets being smaller than in the baryons making up the visible portion of the universe. The same feature also holds for the original theoretical construction [2-5]. However, the difference is that, in the original model [2-5], the quark nuggets are assumed to be absolutely stable at zero pressure, while in the AQN model this stability is achieved by the additional axion domain-wall pressure, see brief review [6] for the details. 
Table 1. Basic properties of the AQNs. The annihilation parameter $\kappa$ is discussed in the text.

\begin{tabular}{cc}
\hline Property & Typical Value or Feature \\
\hline AQN's mass $\left[M_{N}\right]$ & $M_{N} \approx 16 \mathrm{~g}\left(B / 10^{25}\right)[6]$ \\
baryon charge constraints $[B]$ & $3 \times 10^{24} \lesssim B \lesssim 10^{28}(3)$ \\
annihilation cross section $[\sigma]$ & $\sigma \approx \kappa \pi R^{2} \simeq 1.5 \times 10^{-9} \mathrm{~cm}^{2} \cdot \kappa\left(R / 2.2 \times 10^{-5} \mathrm{~cm}\right)^{2}$ \\
density of AQNs $\left[n_{\mathrm{AQN}}\right]$ & $n_{\mathrm{AQN}} \sim 0.3 \times 10^{-25} \mathrm{~cm}^{-3}\left(10^{25} / B\right)[6]$ \\
survival pattern during BBN & $\Delta B / B \ll 1[14,15]$ \\
survival pattern during CMB & $\Delta B / B \ll 1[14,16]$ \\
survival pattern during post-recombination & $\Delta B / B \ll 1[12]$ \\
\hline
\end{tabular}

\section{Acoustic Signals from Meteoroids and AQNs}

\subsection{Blast Wave from Meteoroids}

We start by reviewing a model designed to study meteor-generated infrasound [17]. Originally this model was introduced to describe a blast wave from a lightning discharge, so it has a general character. There are many recent advances in this framework including the comparison with observational data [18,19]. Our goal here is to use this framework to estimate the intensity and frequency characteristics of the infrasound signal generated by AQNs propagating in the atmosphere. Our estimates cannot literally follow [17-19] as the nature of the released energy in the case of AQN is drastically different from the energy sources associated with conventional meteors in the Earth atmosphere. However, we think that the generic scaling features describing the sound waves at large distances hold in both cases. Furthermore, the Mach number $M=v / c_{s} \gg 1$ (here $v$ is the speed of the meteor and $c_{S}$ is the speed of sound) is very large for meteors as well as for AQNs such that cylindrical symmetry is assumed to hold for propagating sound and infrasound waves in both cases.

The basic parameter of the approach [17-19] is the so-called characteristic blast-wave relaxation radius defined as

$$
R_{0} \equiv \sqrt{\frac{E_{l}}{p_{0}}}
$$

where $E_{l}$ is the energy deposited by the meteor per unit trail length, and $p_{0}$ is the hydrostatic atmospheric pressure. The physical meaning of this parameter $R_{0}$ is the distance at which the overpressure approximately equals the hydrostatic atmospheric pressure. In the case of a bomb-like explosion, the relevant parameter can be defined as

$$
R_{1} \equiv \sqrt[3]{\frac{E_{\text {point source }}}{p_{0}}}
$$

where $E_{\text {point source }}$ is the energy deposited to the air as a result of explosion. The parameter $R_{1}$ has the same physical meaning as $R_{0}$ and it determines the distance at which the overpressure approximately equals to the hydrostatic atmospheric pressure.

In simple cases for meteors, the parameter $R_{0}$ can be directly expressed in terms of the Mach number $M$ and the meteor diameter $d_{m}$ as $R_{0} \sim M d_{m}$, see [17-19]. The significance of the parameter $R_{0}$ is that the overpressure $\delta p$ at larger distances can be expressed in terms of dimensionless parameter $x$ defined as follows [17-19]:

$$
\frac{\delta p}{p_{0}}=\frac{2(\gamma+1)}{\gamma} f(x), \quad x \equiv \frac{r}{R_{0}}, \quad f(x \gg 1) \simeq \frac{1}{x^{3 / 4}},
$$

where $\gamma=c_{p} / c_{v}$ is the ratio of the heat capacities. Note that the overpressure $\delta p$ decays faster than $r^{-1 / 2}$ as it would be for a cylindrical sound wave with a given frequency. This is due to increase of the width $l$ of the blast wave packet as follows: $l \sim R_{0} x^{1 / 4}$. Correspondingly, the fundamental sound frequency $v$ decreases as $v \sim c_{S} / l \sim\left(c_{S} / R_{0}\right) x^{-1 / 4}$, 
where $c_{S}$ is the speed of sound. Thus, energy conservation requires faster decrease of the overpressure: $(\delta p)^{2} \propto 1 / V$, where volume of the cylindrical blast wave (for a length $z$ ) is $V \sim 2 \pi r l z \sim 2 \pi R_{0}^{2} z x^{5 / 4}$ (in Equation (8), energy losses are neglected).

The scaling (8) is justified when overpressure is relatively small and geometrical acoustics becomes valid. In case of conventional meteors, all parameters such as $R_{0}$ can be modelled and compared with observations $[18,19]$. We do not have such luxury in the case of AQNs. However, some theoretical estimates can be made, which is the topic of this section.

\subsection{AQN in the Atmosphere}

First, we estimate the parameter $E_{l}$ entering (6). Annihilation of one nucleon on an AQN brings an energy of $2 \mathrm{GeV}$. We should multiply this energy by the number of nucleons which hit the AQN cross section $\sigma=\pi R^{2}$ over a length $l$. The corresponding mass in this volume is $M=\pi R^{2} l \rho$, where the mass density $\rho$ is related to the number density of nucleons in this volume $n_{\text {air }}$ as $\rho=n_{\text {air }} m_{p}$. Note that this relation does not depend on the composition of the gas since we express the result in terms of the proton mass $m_{p}$ rather than atomic mass. It does not depend on the velocity of the nugget as the annihilation energy depends on mass $M c^{2}$, not the velocity (which is the case for meteorites). Thus, the total number of nucleons in this volume is $\pi R^{2} l n_{\text {air }}$ and the energy of annihilation events occurring per unit length while the nugget traverses the atmosphere is:

$$
E_{l} \simeq \kappa \cdot\left(\pi R^{2}\right) \cdot(2 \mathrm{GeV}) \cdot n_{\mathrm{air}} \simeq 10^{4} \cdot \kappa\left(\frac{B}{10^{25}}\right)^{2 / 3}\left(\frac{n_{\mathrm{air}}}{10^{21} \mathrm{~cm}^{-3}}\right) \frac{\mathrm{J}}{\mathrm{m}},
$$

where we translated the $\mathrm{GeV}$ energy in terms of $\mathrm{J}$ as $\mathrm{GeV}=1.6 \times 10^{-10} \mathrm{~J}$. The $n_{\text {air }}$ in this formula is the total number of nucleons in atoms such that $\rho_{\text {air }} \approx n_{\text {air }} m_{p}$. The parameter $\kappa$ as explained above and in Appendix B is introduced to account for the fact that not all matter striking the nugget will annihilate and not all of the energy released by an annihilation will be thermalized in the nuggets (for example, some portion of the energy will be released in the form of axions and neutrinos), see the discussion after Equation(A9). Therefore, $\kappa$ encodes a large number of complex processes including the probability that not all atoms and molecules may be able to penetrate into the color superconducting phase of the nugget to get annihilated. The parameter $\kappa$ could become larger than one in case of the strong ionization such as in the solar corona environment as discussed in [11]. This parameter also includes complicated dynamics due to the very large Mach number $M=v_{\mathrm{AQN}} / c_{s} \gg 10^{2}$ when shock waves are formed and turbulence develops. Both phenomena lead to efficient energy exchange between the nugget and surrounding material. Assuming $10 \mathrm{~km}$ as a typical length scale where emission occurs, one can estimate the total released energy in the atmosphere at the level of $10^{8} \mathrm{~J}$, which represents a small fraction $\sim 10^{-7}$ of the total energy contained in an AQN. In this numerical estimate we assume air mass density $\rho$ averaged over $10 \mathrm{~km}$ height. For simplicity, we keep $\kappa \simeq 1$ in our order-of-magnitude estimates which follow. Directly using the estimate (9), one arrives at the following approximate expression for the parameter $R_{0}$ :

$$
R_{0}^{\mathrm{AQN}} \equiv \sqrt{\frac{E_{l}}{p_{0}}} \sim 0.3\left(\frac{B}{10^{25}}\right)^{\frac{1}{3}}\left(\frac{n_{\mathrm{air}}}{10^{21} \mathrm{~cm}^{-3}}\right)^{\frac{1}{2}} \mathrm{~m} .
$$

Several comments are in order regarding this estimate. In the case of conventional or nuclear explosions, the blast occurs as a result of the interaction of radiation with surrounding material which rapidly heats the material. This causes vaporization of the material, in turn resulting in its rapid expansion, which eventually contributes to formation of the shock-wave. All these effects occurring in conventional explosions at spatial scales much smaller than a typical radius where over-pressure approximately equals to atmospheric pressure. In case of cylindrical symmetry, the relevant parameter is determined by $R_{0}$ in 
Equation (6). In case of a point-like explosion, the corresponding distance $R_{1}$ is determined by (7) which plays the role of $R_{0}$ in this case.

Now we estimate the distances where the radiation is effectively converted to the shock-wave energy. In the case of conventional or nuclear explosions, the dominant portion of the radiation comes in the $20 \mathrm{eV}$ energy range and above. At this energy, the dominant process is the atomic photoeffect with a cross section $\sigma_{\text {p.e. }} \sim 10^{7}$ barn and higher, such that the photon attenuation length $\lambda \sim 10^{-6} \mathrm{~g} / \mathrm{cm}^{2}$, see, for example, Figure 33.15 and Figure 33.19 in [20] and references therein. In case of meteoroids, the emission normally occurs in the 1 to $20 \mathrm{eV}$ range, which includes visible light. These spectral features in air imply that the energy due to heating is completely absorbed on spatial scales much shorter than $R_{0}$ defined by (6), i.e.,

$$
\frac{\lambda}{\rho_{\text {air }}} \lesssim 10^{-3} \mathrm{~cm} \ll R_{0} \quad[\text { meteoroids }] .
$$

This should be contrasted with the AQN case with a drastically different radiation spectrum with typical energy in the $\sim 20-40 \mathrm{keV}$ range as reviewed in Appendix B. Atomic photoelectric effect is still the dominant process for this energy band and the photon attenuation length is $\lambda \sim 0.5 \mathrm{~g} / \mathrm{cm}^{2}$, so that

$$
L=\frac{\lambda}{\rho_{\text {air }}} \simeq 5 \mathrm{~m} \gg R_{0}^{\mathrm{AQN}} \quad[\mathrm{AQN} \text { events }] .
$$

These estimates suggest that only a small portion of the energy (9) will be released in the form of a blast, while the rest of the energy will heat the surrounding material. The attenuation length is even longer for higher-energy photons which saturate the total intensity for $T_{\mathrm{AQN}} \simeq 40 \mathrm{keV}$, see Appendix B. One should emphasize that the difference in the spectrum dramatically modifies the properties of the acoustic blast as discussed above. One should also mention that the collisions between atmospheric molecules and the AQN may generate radiation at much lower frequency bands, including visible light, which can potentially observed. However, we expect that the total intensity for such emissions is suppressed in comparison with X-ray direct emission from AQN.

We can do an estimate of overpressure in this case as follows. The annihilation energy $E_{l} z$ released on a track of length $z$ is absorbed in the volume of the cylinder $V=\pi L^{2} z$. The internal energy of a diatomic ideal gas (air) is given by $U=(5 / 2) P V$. This gives an estimate of overpressure inside this volume $V$ :

$$
\delta p_{0} \approx \frac{E_{l}}{2.5 \pi L^{2}} \quad[\mathrm{AQN} \text { events }] .
$$

As mentioned above, outside this volume, $\delta p$ decreases as $f(\bar{x}) \approx \bar{x}^{-3 / 4}$, where we introduced the dimensionless parameter $\bar{x}=r / L$, which plays the same role as $x$ in the formula for meteoroids (8):

$$
\delta p(r) \approx \frac{\delta p_{0}}{\bar{x}^{3 / 4}} \approx \frac{E_{l}}{2.5 \pi L^{2}}\left(\frac{1}{\bar{x}}\right)^{3 / 4} \quad[\text { AQN events }] .
$$

To illustrate the significance of the estimate (14), we present an order-of-magnitude numerical estimate for the overpressure at a distance $r$, with the annihilation energy given by Equation (9) and absorption of this energy within the radius $L=5 \mathrm{~m}$ as estimated by (12):

$$
\delta p \approx 0.03 \mathrm{~Pa}\left(\frac{B}{10^{25}}\right)^{2 / 3}\left(\frac{100 \mathrm{~km}}{r}\right)^{3 / 4}[\mathrm{AQN} \text { events }] .
$$

This estimate shows that a typical AQN generates a small overpressure $\delta p / p \sim 10^{-3}$ even inside the absorption region $r \lesssim L$, which should be contrasted with the meteoroid case (8) 
where $\delta p / p \approx 1$ at $r \simeq R_{0}$. The difference is due to the large length $L \simeq 5 \mathrm{~m}$ in comparison with the small absorption distance $\sim 10^{-3} \mathrm{~cm}$ for the meteoroids (11). The temperature increase in surrounding region $\delta T / T \sim \delta p / p \sim 10^{-3} \ll 1$ is too small to produce visible thermal radiation around the AQN path. This temperature should not be confused with the much higher internal AQN temperature $T_{\mathrm{AQN}} \sim 10 \mathrm{keV}$.

Another important characteristic of the acoustic waves produced by meteoroids is the scaling behaviour of the so called line-source wave period $\tau(x)$ at large distances. The scaling behaviour can be expressed in terms of the same dimensionless parameter $x$ introduced above, and it is given by [17-19]:

$$
\tau(x) \simeq 0.562 \tau_{0} x^{1 / 4}, \tau_{0}=2.81 \frac{R_{0}}{c_{s}} \text { [meteoroids] },
$$

where $\tau_{0}$ is the so-called fundamental period where numerical factors 2.81 and 0.562 in Equation (16) have been fitted from the observations [19]. Equation (16) determines the frequency of the sound (infrasound) wave at a distant point $x$ :

$$
v(x) \equiv \tau^{-1}(x) \sim \tau_{0}^{-1} x^{-1 / 4}, x \gg 1 \text { [meteoroids] }
$$

The same scaling behaviour is expected to hold for the AQN case. However, the parameters for AQNs are different:

$$
\tau_{0} \sim \frac{L}{c_{s}}, \tau(\bar{x}) \sim \tau_{0} \bar{x}^{1 / 4}, v_{0} \equiv \frac{1}{\tau_{0}} \sim 70 \mathrm{~Hz},
$$

where we ignore all numerical factors which in the case of meteoroids were fixed by matching with observations, and obviously cannot be applied to our present studies of the AQNs. In this case we arrive at the following estimate for the frequency at a distance $r$ :

$$
v(\bar{x}) \sim \frac{v_{0}}{\bar{x}^{1 / 4}} \sim 6 \mathrm{~Hz}\left(\frac{100 \mathrm{~km}}{r}\right)^{1 / 4}[\text { AQN events }] .
$$

It is hard to estimate the accuracy of our results because of the complexity of the interaction of the AQN with the environment when Mach number $M \equiv\left(v_{\mathrm{AQN}} / c_{s}\right) \gg 1$. We think that the largest uncertainty is related to the coefficient $\kappa$ entering (9). As the $\delta p \propto E_{l}$ according to (13) we expect an order of magnitude uncertainty for $\delta p$, similar to $\kappa$. At the same time the uncertainty for $v_{0} \propto L^{-1}$ is much smaller (factor of two or so) because it is determined by parameter $L$ defined by (12) which is known with much better accuracy. In case of interaction of the AQN with the rocks or water, discussed in the next subsection, the uncertainties are much higher, as we argue below.

Thus, at a large distance from the AQN track in the air there will be emission of low frequency infrasound waves. We will see below that for the signal from an underground AQN track, the overpressure and the frequency are both several orders of magnitude higher.

\subsection{AQN Propagating Underground}

One should emphasize that the infrasound waves originating from AQNs as estimated in Section 3.2 will be always accompanied by sound waves emitted by the same AQNs when the nuggets hit the Earth surface and continue to propagate in the deep underground (solid rocks and water). The corresponding estimates of intensity and frequency of the sound emitted as a result of the annihilation events occurring underground are presented in this subsection. Our main intention in this subsection is to provide the corresponding estimates. A hope is that the acoustic wave propagating on the surface can be detected by a different instrument (see e.g., Distributed Acoustic Sensing (DAS) technology in the next section) along with infra-sound signal. The synchronized detection of these very different signals may be the key element to study such kind of events. All estimates in 
this subsection are inevitably very crude (due to a large number of unknown parameters entering the formulae) and presented here exclusively for the illustrative purposes.

The starting point is similar to (9) which for underground rocks assumes the form:

$$
E_{l}^{\downarrow} \simeq \kappa \cdot\left(\pi R^{2}\right) \cdot(2 \mathrm{GeV}) \cdot n_{\text {rock }} \simeq 10^{7} \cdot \kappa\left(\frac{B}{10^{25}}\right)^{2 / 3}\left(\frac{n_{\text {rock }}}{10^{24} \mathrm{~cm}^{-3}}\right) \frac{\mathrm{J}}{\mathrm{m}^{\prime}}
$$

where we use the notation $E_{l}^{\downarrow}$ for the energy produced by annihilation (some of which may remain in the AQN) to avoid confusion with the similar Equation (9) applied to the atmosphere, $n_{\text {rock }}$ is the total number of nucleons in atoms such that $\rho=n_{\text {rock }} m_{p}$.

We introduce an unknown parameter $\xi^{\downarrow}$ which applies to the underground case (at sufficiently high density of the surrounding material) to account for the complicated physics which describes the transfer of the AQN energy into the the surrounding material energy denoted as $E_{\text {blast }}^{\downarrow}$ :

$$
E_{\text {blast }}^{\downarrow}=10^{7} \cdot \xi^{\downarrow}\left(\frac{B}{10^{25}}\right)^{2 / 3}\left(\frac{n_{\text {rock }}}{10^{24} \mathrm{~cm}^{-3}}\right) \frac{\mathrm{J}}{\mathrm{m}} .
$$

There are several important new elements in comparison with the atmospheric case discussed in Section 3.2. First of all, the increase of the density of the surrounding material naively drastically increases the released annihilation energy as Equation (20) suggests, assuming that the coefficient $\mathcal{K}$ remains the same as in the atmospheric case, Equation (9). However, it is expected that this assumption is strongly violated. The main reason for that is due to increase of the internal temperature $T_{\mathrm{AQN}}$ which consequently leads to strong ionization of the positrons from electrosphere. As a result of this ionization the positron density of the electrosphere (which is responsible for the emissivity) drastically decreases. It suppress the emissivity from the electrosphere as Equation (A4) states. If one removes the low-energy positrons from the electrosphere, the suppression factor could be $\xi^{\downarrow} \sim 10^{-2}$ and even much smaller.

The second important effect which was ignored in the atmosphere in Section 3.2 is that $\delta p$ could be much larger underground in comparison with the estimate of Equation (15). It results in pushing material from the AQN path which effectively decreases the geometrical cross section $\pi R^{2}$ assumed in (20). This effect further suppresses the parameter $\xi^{\downarrow}$ entering (21).

We cannot at the moment compute $\xi^{\downarrow}$ from first principles. The consistent procedure would be a mean-field computation of the positron density by imposing the proper boundary conditions relevant for nonzero temperature and non-zero charge, similar to $T_{\mathrm{AQN}} \approx 0$ computations carried out in [21,22]. The corresponding computations have not been done yet, and we keep the parameter $\xi^{\downarrow}\left(T_{\mathrm{AQN}}\right)$ as a phenomenological free parameter. Therefore, we keep it as a phenomenological unknown parameter which strongly depends on the environment, temperature $T_{\mathrm{AQN}}$ and many complex processes as mentioned above.

Another important parameter is the absorption length $L^{\downarrow}\left(T_{A Q N}\right)$ for the energy emitted by AQN in underground (hence the $\downarrow$ label), which also indirectly depends on the AQN internal temperature $T_{A Q N}$. This is because the length $L^{\downarrow}$ strongly depends on the energy of the photons emitted by the AQNs, which is determined by the internal temperature $T_{A Q N}$. For the photon energy $\sim 100 \mathrm{keV}$, an absorption length in silicon is about $L^{\downarrow} \simeq 2 \mathrm{~cm}$. However, it is an order of magnitude larger for $1 \mathrm{MeV}$ photons. We account for this uncertainty by introducing another unknown dimensionless parameter $\eta$ defined as follows: $L^{\downarrow}\left(T_{A Q N}\right)=(2 \mathrm{~cm}) \cdot \eta$. In terms of these unknown parameters the deposited energy per unit volume $\epsilon_{\text {blast }}$ surrounding the $\mathrm{AQN}$ can be estimated as follows:

$$
\epsilon_{\text {blast }} \simeq 10^{2} \frac{\mathrm{J}}{\mathrm{cm}^{3}} \cdot\left(\frac{\xi^{\downarrow}}{10^{-2}}\right) \cdot\left(\frac{1}{\eta^{2}}\right)
$$


which leads to an instantaneous increase of temperature $\delta T$ of the surrounding material:

$$
\delta T \simeq 10^{2} K \cdot\left(\frac{\xi^{\downarrow}}{10^{-2}}\right) \cdot\left(\frac{1}{\eta^{2}}\right)
$$

In this estimate we assume an average heat capacity of a rock $\sim 2000 \mathrm{~J} / \mathrm{kg} \mathrm{K}$ and density $\sim 2 \mathrm{~g} / \mathrm{cm}^{3}$. We now in position to estimate the overpressure for the blast wave in two different approximations. First, we may estimate the overpressure as deposited energy per unit volume. This yields

$$
\delta p \simeq 10^{7} \mathrm{~Pa} \cdot\left(\frac{\xi^{\downarrow}}{10^{-2}}\right) \cdot\left(\frac{1}{\eta^{2}}\right) \quad \text { at } \quad r \simeq L^{\downarrow} .
$$

Another approximation is based on an increase of pressure due to the thermal expansion of solids. Relative thermal expansion of a rock $\delta x / x \sim 10^{-5} / \mathrm{K}$, the Young modulus is $\sim 10 \mathrm{GPa} \sim 10^{10} \mathrm{~Pa}$. This gives the same order of magnitude as in the dimensional estimate (23).

Our next task is to estimate the amplitude of the wave at large distance $r$. Using the conventional scaling arguments when $\delta p(r) \sim 1 /\left(\bar{x}^{\downarrow}\right)^{3 / 4}$ with dimensionless parameter $\bar{x}^{\downarrow}$ defined as $\bar{x}^{\downarrow}=r / L^{\downarrow}$ we arrive to the following estimate for overpressure at a distance $r$ :

$$
\delta p(r) \sim 10^{2} \cdot\left(\frac{\xi^{\downarrow}}{10^{-2}}\right) \cdot\left(\frac{1}{\eta}\right)^{\frac{5}{4}} \cdot\left(\frac{100 \mathrm{~km}}{r}\right)^{\frac{3}{4}} \mathrm{~Pa} .
$$

Following the same logic as for Equation (19) we obtain a numerical estimate for the frequency of sound emitted by an AQN propagating underground:

$$
\tau_{0} \sim \frac{L^{\downarrow}}{c_{S}}, \tau\left(\bar{x}^{\downarrow}\right) \sim \tau_{0} \cdot \sqrt[4]{\bar{x}^{\downarrow}}, \quad v_{0} \equiv \frac{1}{\tau_{0}} \simeq 170\left(\frac{1}{\eta}\right) \mathrm{kHz},
$$

where we use $c_{s} \simeq 3 \mathrm{~km} / \mathrm{s}$ for speed of sound in rocks. For large distances $r$ our estimate for the frequency becomes

$$
v\left(\bar{x}^{\downarrow}\right) \sim \frac{v_{0}}{\sqrt[4]{\bar{x}^{\downarrow}}} \sim 3.5\left(\frac{1}{\eta}\right)^{\frac{3}{4}} \cdot\left(\frac{100 \mathrm{~km}}{r}\right)^{\frac{1}{4}} \mathrm{kHz}, \quad \text { [estimates for rocks] }
$$

which is almost 3 orders of magnitude higher than the frequency of the infrasound emitted by AQNs in atmosphere (19).

In the estimate (24) above we assumed that the absorption of sound can be neglected. In an analogous estimate studied in previous Section 3.2 for the infrasound produced by AQN in the air this assumption is well- justified. One can easily convince oneself that the estimate (15) is practically unaffected by absorption on the distance well above $100 \mathrm{~km}$. This assumption is justified for water as estimated below. However, in sedimentary rocks the absorption may be significant [23] and could modify the estimates given for water below. Propagation of waves inside solid earth is a complicated phenomenon. It may be sufficient to say that there are different types of waves (longitudinal, transverse, and surface waves) which have different speed and absorption properties, they also propagate in different environments. This means that a signal at a detector may have more than one maximum. To simplify our estimates we consider here the propagation of the waves in water which looks like a simpler problem and provides a good example.

To proceed with estimates we note that in water the sound absorption length scales as $\propto v^{-2}$. This scaling is a very generic feature of any fluid when the absorption coefficient is expressed in terms of the viscosity and thermal conductivity, see e.g., [24]. A proper estimation of the absorption effects must include the integration over distance where sound wave propagates since the frequency depends on the distance according to (26) as $v \propto r^{-1 / 4}$ and the absorption length $l \propto v^{-2} \propto r^{1 / 2}$. Using these relations and equations 
for frequency (25), (26) we integrate absorption over distance $r$. As a result, Formula (24) will receive an additional exponential factor which describes the suppression of the sound intensity (intensity $\propto \delta p^{2}$ ) due to the absorption of the sound wave:

$$
\exp (-X), \text { where } X \approx 2 \frac{\left[L^{\downarrow} r\right]^{\frac{1}{2}}}{l_{0} \eta^{2}}
$$

where $l_{0}$ is the absorption length for the initial frequency $v_{0}$. For a numerical estimate of the blast wave absorption we may use detailed data on the sound absorption in sea water [25]. The radiation absorption length for energy $\sim 100 \mathrm{keV}$ in water is $4.15 \mathrm{~cm}$, so we assume $L^{\downarrow}=4.15 \eta \mathrm{cm}$. The speed of sound in water is $c_{S}=1.5 \mathrm{~km} / \mathrm{s}$, so we have our estimate for frequency in water $v_{0} \simeq 36 \mathrm{KHz}$ and

$$
v\left(\bar{x}^{\downarrow}\right) \sim \frac{v_{0}}{\sqrt[4]{\bar{x}^{\downarrow}}} \sim\left(\frac{1}{\eta}\right)^{\frac{3}{4}} \cdot\left(\frac{100 \mathrm{~km}}{r}\right)^{\frac{1}{4}} \mathrm{kHz}, \quad \text { [estimates for water] }
$$

The sound absorption length in water is $l_{0} \simeq 0.45 \mathrm{~km} \mathrm{[25]} \mathrm{and} X \simeq 0.2 \eta^{-3 / 2}$ for $r=100 \mathrm{~km}$. Thus, the absorption of the blast wave in water is insignificant. It may be a dramatically different conclusion for sedimentary rocks when the absorption coefficient is significantly larger and frequency dependence is linear $\sim v$ [23] rather than quadratic $v^{2}$ in case of water [24]. One should not confuse the estimation for water (28) with our estimates for rock (26) where all parameters are slightly different. We consider these two cases separately because the absorption in water can be ignored in our estimates, in contrast with the case of rocks.

We conclude this subsection with the following remarks. First of all, in case of conventional meteoroids all numerical factors entering the scaling relations such as (8) and (16) have been fitted to match with numerous observations. It should be contrasted with our studies where there is no such luxury with observed and measured events. As a result we introduce into our AQN estimates empirical parameters $\xi^{\downarrow}$ and $\eta$ which are very hard to compute from the first principles, but could be fixed by future observations. Further studies are needed to collect more statistics of mysterious events when sound signatures are recorded without any traces in the synchronized optical monitoring systems.

We summarize this section with some important comments. Our prediction for the overpressure (15) for a typical AQN event with $B \simeq 10^{25}$ at the infrasound frequency (19) suggests that the existing instruments such as ELFO are not sufficiently sensitive to detect such small signals on the level of $\delta p \simeq 0.03 \mathrm{~Pa}$. Nevertheless, some strong and rare events still can be recorded with existing technology. In particular, it is tempting to identify a single mysterious event recorded by ELFO [7] as the AQN event with very large baryon charge $B \simeq 10^{27}$, see Appendix A with detailed arguments. Here we just highlight this reasoning.

The radius of the nugget scales as $R \sim B^{1 / 3}$, which effectively leads to an increase in the number of annihilation events for larger nuggets, which eventually releases higher energy output per unit length as Equation (9) states. Therefore, the intensity of the event scales correspondingly. The powerful explosion recorded by ELFO with overpressure on the level $\delta p \simeq 0.3 \mathrm{~Pa}$ is consistent with the AQN annihilation event with large $B \simeq 10^{27}$. Such intense events are relatively rare ones according to (4) and (5) as the frequency of appearance is proportional to $f(B) \sim B^{-\alpha}$ with $\alpha \simeq(2-2.5)$. It might be a part of an explanation of why this area has observed a single event in 10 years rather than observing similar events much more often.

It is obvious that we need much more statistics for systematic studies of relatively small but frequent typical events with $B \simeq 10^{25}$. In the next section we present a possible design of an instrument which could be sufficiently sensitive to infrasound and seismic signals to fulfill this goal. If our proposal turns out to be successful, it will be possible to routinely record a large number of such events which manifest themselves in the form of the infrasonic and seismic signals, while the optical synchronized cameras may not see any light from these events. Infrasonic signals must be always accompanied by sound 
and seismic waves as discussed above, which can be routinely recorded by conventional seismic stations. These events should demonstrate the daily and annual modulations as the source for these events is the dark matter galactic wind.

\section{Detection Strategy and Possible Instruments}

As mentioned in the previous section, the sensitivity of instruments similar to ELFO is insufficient to record relatively small events in the atmosphere which occur approximately once a day in an area of $(100 \mathrm{~km})^{2}$ with $\delta p \simeq 0.01 \mathrm{~Pa}$ and frequency $v \simeq 5 \mathrm{~Hz}$. In this section, we suggest several possible designs of instruments which maybe sufficiently sensitive to detect signals from common small AQNs with $B \sim 10^{25}$. One should emphasize that the present section should be considered a proposal for future studies where different approaches are being advocated. At this point, we do not know if any of these ideas can be realized in practise. However, we think these new ideas should be explicitly stated, so that researchers from various communities could consider searching for AQN dark matter.

We start the overview with a promising recent development, Distributed Acoustic Sensing (DAS), which is becoming a conventional tool for seismic and other applications, see, for example, [26-29] and references therein. The basic idea of these activities can be explained as follows. It has been known for quite sometime that distributed optical fiber sensors are capable of measuring the signals at thousands of points simultaneously using an unmodified optical fiber as the sensing element. The recent development is that the DAS is capable of measuring strain changes at all points along the optical fiber at acoustic frequencies, which is crucial for our studies of the acoustic waves emitted due to the AQN passage.

The main element of the DAS technology is that a pulse of light is sent into optical fiber and, through scattering in the glass, a small amount of the incident light is scattered back towards the sensing unit. The key point is that the DAS is capable of determining from this scattered light, a component which indicates changes in the local axial strain along the fibre. It has been shown that this technology is capable of detecting signals at frequencies as low as $8 \mathrm{mHz}$ and as high as $49.5 \mathrm{kHz}$ with sensitivity at the level of $\delta p \sim 0.1 \mathrm{mBar} \approx 10 \mathrm{~Pa}$ [26] which is more than sufficient for our purposes for $\delta p$ as estimated in (24) and typical frequencies in $\mathrm{kHz}$ band as estimated in (26). Furthermore, it has been shown that using an amplifier chain one can extend the range of DAS units to $82 \mathrm{~km}$, while maintaining high signal quality [26]. Such a long range on the scale of $100 \mathrm{~km}$ matches well with what is needed for AQN-passage detection. Indeed, we anticipate approximately one event per day per an area of $(100 \mathrm{~km})^{2}$ according to (5) reviewed in Section 2. An important point is that such studies can in principle detect not only the intensity and the frequency of the sound wave, but also the direction of the source. We note that networks of optical-fiber telecommunication cables cover a significant part of the Earth's surface.

We anticipate that the main problem with DAS will be separation of the AQN signal from the seismic noise and numerous spurious events. We discuss one of the possibilities to separate the signal from a much larger noise below. The main point is that the AQN signal must show the annual (29) and daily (30) modulations characteristic of the dark matter galactic wind, in contrast with much more numerous and much more intense random events.

The phase of an optical wave propagating over a distance in a fiber accumulates effects of external perturbation and is highly sensitive to these perturbations. The changes of the phases are detected by a network of synchronised atomic clocks and optical cavities. The original idea was to detect transient effects produced by clumps of low-mass dark matter and by passing topological defects. Here, we suggest that such networks may also be sensitive to the blast waves produced by AQN.

The blast wave pressure $\delta p$ produces a minor change of the optical fiber index of refraction and changes the optical length, $\delta L_{\mathrm{opt}}$. This leads to a change in the phase of order $\phi=\delta L_{\text {opt }} / \lambda$, where the wavelength $\lambda \sim 500 \mathrm{~nm}=0.5 \times 10^{-6} \mathrm{~m}$. The relative accuracy of the best atomic clocks has surpassed $10^{-18}$, but this requires a long observation time. As an 
estimate of the best current sensitivity we may take $\delta L \sim 10^{-19} \mathrm{~m}$ as in LIGO and VIRGO gravitational wave detectors. This very high sensitivity indicates that the main problem here is the separation of the AQN signal from the seismic noise. One of possibilities to separate the signal from a much larger noise was suggested in [30].

The basic idea of [30] can be explained as follows. The AQN flux is given by (5) if averaged over very long period of time, longer than a year. However, due to the relative motion and orienation of the Sun, Earth and the galaxy, the AQN flux, as that of any other dark mater particle, receives the time-dependent factor $A_{(a)}(t)$ representing the annual modulation which is defined as follows:

$$
A_{(\mathrm{a})}(t) \equiv\left[1+\kappa_{(\mathrm{a})} \cos \Omega_{a}\left(t-t_{0}\right)\right],
$$

where $\Omega_{a}=2 \pi \mathrm{yr}^{-1} \approx 2 \pi \cdot 32 \mathrm{nHz}$ is the angular frequency of the annual modulation and label " $a$ " in $\Omega_{a}$ stands for annual. The $\Omega_{a} t_{0}$ is the phase shift corresponding to the maximum on 1 June and minimum on 1 December for the standard galactic DM distribution, see [31,32]. Similar daily modulations are also known to occur [33] and can be represented as follows:

$$
A_{(\mathrm{d})}(t) \equiv\left[1+\kappa_{(\mathrm{d})} \cos \left(\Omega_{d} t-\phi_{0}\right)\right],
$$

where $\Omega_{d}=2 \pi$ day $^{-1} \approx 2 \pi \cdot 11.6 \mu \mathrm{Hz}$ is the angular frequency of the daily modulation, while $\phi_{0}$ is the phase shift similar to $\Omega_{a} t_{0}$ in (29). It can be assumed to be constant on the scale of days. However, it actually slowly changes with time due to the variation of the direction of DM wind with respect to the Earth. The modulation coefficients $\kappa_{(\mathrm{a})}$ and $\kappa_{(\mathrm{d})}$ have been computed in the AQN model in [33].

The idea advocated in [30] is to fit the data to the modulation Formulae (29) and (30), even if the noise is large and exceeds the expected signal. The key point here is the statistics factor and accumulation of the signal for a long period of time assuming that the noise can be treated as being random in contrast with signal being characterized by well defined frequencies $\Omega_{a}$ and $\Omega_{d}$. A hope is to discover the annual (29) and daily (30) modulations by recording a large number of $\mathrm{AQN}$ events which represent the dark matter galactic wind in this specific model.

This idea of separation of the signal from the spurious effects should work even if the noise also has the same periodicity of $24 \mathrm{~h}$ such as the temperature variation. The point is that the phase shift $\phi_{0}$ of the signal and the temperature variation do not coincide. Furthermore, one could study the variation of the phase shift $\phi_{0}$ within one season to discriminate the genuine signals from the noise as the DM signal slowly drifts with the season while the noise (such as temperature) does not.

Another way to discriminate the signal from the noise is to study the synchronized signals and time delays by different detectors from a single AQN propagating in atmosphere. This approach may also reconstruct the AQN's trajectory as argued in [34].

A specific signal from $A Q N$ tracks is very different from the ordinary seismic noise and earthquakes. Therefore, AQN signals may, in principle, be detected by an existing network of seismic stations. In addition to optical-fiber based methods, it may be possible to search for the AQN-passage signatures also in the large volumes of existing historical data from networks of seismometers [35].

We would like to briefly mention other possibilities for the AQN detection, see also relevant references in Section 2 leading to the constraints (3). The AQN produce only a small amount of visible light as we already mentioned. However, the emitted X-rays will be absorbed and heat the atmosphere along the track on scales of order $L$ as Equation (12) suggests. It may produce vapor tracks along the AQN path.Therefore, one may try to observe infrared radiation from AQN tracks using infrared telescopes being synchronized with infrasonic detectors and all-sky cameras. Similarly, the AQN tracks also produce microwave and radio wave radiation which may be detected by radio telescopes which can be also synchronized with infrasonic and all sky cameras. 
Furthermore, while an AQN itself is only $R \simeq 10^{-5} \mathrm{~cm}$ in size, nevertheless, it may leave larger and noticeable cracks along its path in solids as instantaneous defect creation and temperature increase occur on a cm scale according to (22). The cracks could be sufficiently large to be observed. A search for AQN annihilation tracks could also be performed in old rocks and Antarctic ice.

Finally, one more possibility to study the long ranged signals which could be produced by the AQN traversing the Earth atmosphere is to search for signals similar to the ones which are normally attributed to the ultra high energy cosmic rays. In fact, the recent unusual mysterious bursts recorded by the Telescope Array Experiment might be precisely those types of events as argued in [36]. The very puzzling multi-modal clustering events recorded by the HORIZON 10T collaboration may be also related to the AQN traversing the atmosphere as argued in [37].

\section{Conclusions}

The main results of the present work can be summarized as follows:

1. We argue that an AQN propagating in the Earth's atmosphere generates infrasonic waves. We estimated the intensity (15) and spectral features (19) of these waves for a typical AQN event with $B \simeq 10^{25}$;

2. We also performed similar estimates for an AQN propagating inside the Earth;

3. We propose a detection strategy to search for a signal generated by a typical relatively small $A Q N$ event with $B \simeq 10^{25}$ by using distributed acoustic sensing as existing instruments are not sufficiently sensitive to detect such signals;

4. Specific signals from $A Q N$ passage may also be detected with a variety of alternative techniques, for instance, with an existing network of seismic stations (or even by analyzing the already existing data) or by analyzing specific synchronized events which may mimic the Ultrahigh Energy Cosmic Rays, as mentioned at the end of Section 4;

5. We further speculate that the mysterious explosion which occurred on 31 July 2008 and which was properly recorded by the dedicated Elginfield Infrasound Array [7] might be a good candidate for an $\mathrm{AQN}$-annihilation event with a large $B \simeq 10^{27}$ as our basic estimates for the overpressure (A1) and the frequency (A2) are tantalizingly close to the signal recorded by ELFO.

One should emphasize that our estimates are based on the parameters of the AQN model which were fixed long ago for completely different reasons, namely, to explain various different phenomena occurring in drastically different environments, as reviewed in Section 2.

Why should one take this (AQN) model seriously? A simple answer is as follows. Originally, this model was invented to explain the observed relation $\Omega_{\mathrm{DM}} \sim \Omega_{\text {visible }}$ where the "baryogenesis" framework is replaced with a "charge-separation" paradigm, as reviewed in the Introduction and in Section 2. This model is shown to be consistent with all available cosmological, astrophysical, satellite, and ground-based constraints, where AQNs could leave a detectable electromagnetic signature with one and the same set of parameters. Furthermore, it was shown that AQNs could have been formed and could have survived the harsh environment of the early universe. Therefore, AQNs deserve to be considered a viable DM candidate. Finally, the same AQN framework may also explain a number of other (naively unrelated) observed phenomena as discussed in a recent review [6].

Author Contributions: Conceptualization: D.B., V.V.F. and A.Z.; methodology and formal analysis: V.V.F. and A.Z; original draft preparation and writing, review, editing, D.B., V.V.F. and A.Z. All authors have read and agreed to the published version of the manuscript. 
Funding: The work of D.B. was supported in part by the DFG Project ID 390831469: EXC 2118 (PRISMA+ Cluster of Excellence). D.B. also received support from the European Research Council (ERC) under the European Union Horizon 2020 Research and Innovation Program (grant agreement No. 695405), from the DFG Reinhart Koselleck Project and the Heising-Simons Foundation. The work of V.V.F. is supported by the Australian Research Council and the Gutenberg Fellowship. The work of A.Z. was supported in part by the National Science and Engineering Research Council of Canada.

Data Availability Statement: In Appendix A we used several links describing the mysterious explosion which occurred on 31 July 2008 and which was properly recorded by dedicated Elginfield Infrasound Array as shown on Figures A1-A3. These Figures were adopted from https:/ / aquarid. physics.uwo.ca/research/infrasound/is_mysteriousexplosions.html [7]. The data for sound absorption coefficients have been taken from "Absorption length calculator": http:/ / resource.npl.co.uk/ acoustics/techguides/seaabsorption/ [25]. The information on "Incorporated Research Institutions for Seismology (IRIS) network" is available at https:/ /www.iris.edu/hq/programs/gsn [35]. All data being used in this work were obtained from these web sites at the moment of submission on 11 January 2022.

Acknowledgments: The authors are grateful to Mark G. Raizen, Vincent Dumont, Nataniel Figueroa Leigh and Jonathan B. Ajo-Franklin for the discussion of fiber-networks for acoustic detection.

Conflicts of Interest: The authors declare no conflict of interest. The funders had no role in the design of study; in the collection, analyses, or interpretation of data; in the writing of the manuscript, or in the decision to publish the results.

\section{Appendix A. Theory Confronts Observations}

As we mentioned in the main text of the paper our prediction for the overpressure (15) for a typical $A Q N$ event with $B \simeq 10^{25}$ at the infrasound frequency (19) suggests that the predicted signal is too weak to detect by existing instruments such as ELFO. We also mentioned that some strong and intense events may still occur and can be recorded with existing technology. However, the frequency of appearance for such intense events should be very low.

In particular, it is tempting to identify a single mysterious event recorded by ELFO [7] as the AQN event with very large baryon charge $B \simeq 10^{27}$.

The goal of this Appendix is to present the arguments suggesting that the mysterious explosion which occurred on 31 July 2008 and which was properly recorded by dedicated Elginfield Infrasound Array [7] is consistent with the AQN annihilation events. Localization of the source position, Elginfield Infrasound Array (ELFO) and seismic stations are shown on Figure A1 adopted from [7].

The sounds, as reported by residents of Kincardine, Ontario, Canada were apparently loud enough to rattle windows and objects on walls. An important point here is that the infrasound detection associated with this sound shock was recorded by ELFO as presented in Figure A2 with a typical overpressure $\delta p \sim 0.3 \mathrm{~Pa}$. These observations (along with non-observations in the all-sky camera network) ruled out a meteor source, as well as operations at the Bruce Nuclear Power Plant, while Goderich salt mine logs eliminated it as a source [7]. Furthermore, a local airport radar reported no aircraft in the area at the time. The impulses were also observed seismically as ground coupled acoustic waves around South Western Ontario and Northern Michigan as shown on Figure A3. One should emphasize that the seismic stations which record ground coupled acoustic waves may detect signals before ELFO because the speed of sound in a solid is much higher than in the atmosphere. Furthermore, the propagation of seismic waves is complicated and depends on the geological structure, which is specific to the local area. There are also different propagation speeds for transverse and longitudinal waves. The analysis of the seismic stations [7] suggests that two blasts (if interpreted as quarry-type explosions) are localized in the area denoted with the red symbols with error bars on Figure A1. It should be contrasted with the infrasound signal detected by ELFO which arrives from the atmosphere from a different location and propagates with a much lower speed. 


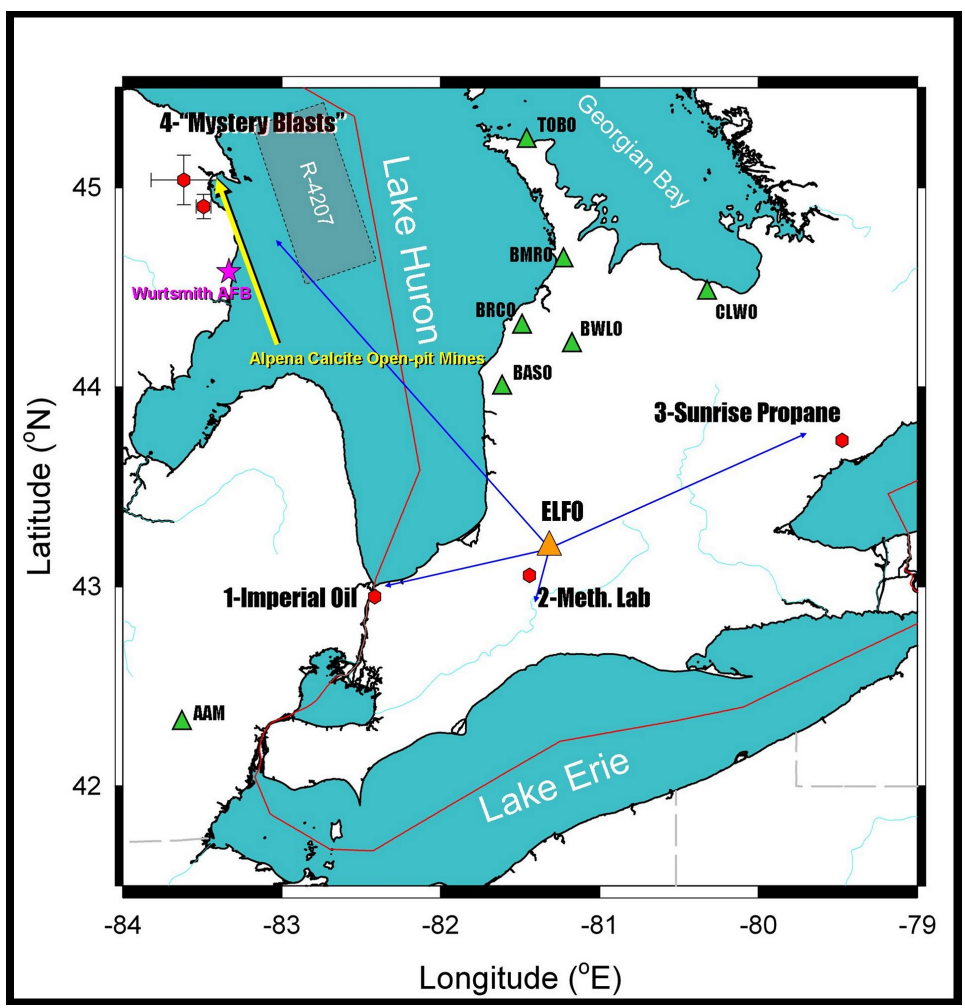

Figure A1. Location of ELFO and seismic stations in the area, adopted from [7], see link in "Data Availability Statement", above the "Acknowledgements". One degree along the latitude corresponds to $112 \mathrm{~km}$. i.e., $1^{0} \approx 112 \mathrm{~km}$, while along the longitude $1^{0} \approx 82 \mathrm{~km}$. It explains our benchmark $300 \mathrm{~km}$ in Equations (A1) and (A2) which covers the relevant area shown on the map. The green triangles represent the seismic stations in the area. Red symbols with error bars represent the position of the blasts (with errors) assuming quarry-type explosions, other red symbols with blue lines directed to them show the directions from ELFO to these potential sources of the explosions (they have been considered but ruled out as the sources).

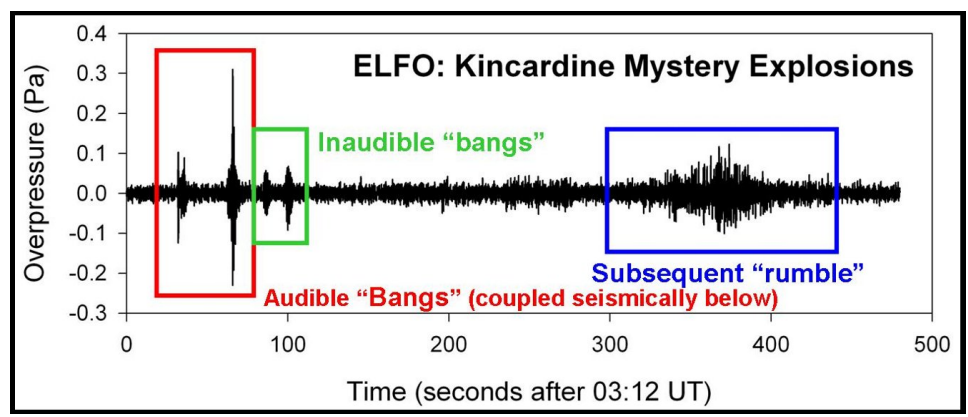

Figure A2. Infrasound impulses as recorded by ELFO, adopted from [7], see link in "Data Availability Statement", above the "Acknowledgements".

Now we are in a position to apply the scaling behaviour (15) to see if the mysterious event recorded by ELFO might have resulted from the AQN annihilation events along its trajectory when it crosses the atmosphere in this area. Our estimate (15) suggests that the overpressure $\delta p$ assumes the following numerical value at $r \simeq 300 \mathrm{~km}$ :

$$
\delta p \approx 0.3 \operatorname{Pa}\left(\frac{B}{10^{27}}\right)^{2 / 3}\left(\frac{300 \mathrm{~km}}{r}\right)^{3 / 4},
$$

where we choose the benchmark for $r=300 \mathrm{~km}$ corresponding to a typical distances in the area shown on Figure $A 1$, and $B=10^{27}$ to bring the numerical coefficient close to 
the measured value $\delta p \simeq 0.3 \mathrm{~Pa}$ as recorded by ELFO, see Figure A2. Estimation for the intensity (A1) is consistent with observation $\delta p \simeq 0.3 \mathrm{~Pa}$ if one assumes that the mysterious explosion had resulted from the AQN annihilation event of a relatively large size with the baryon charge $B \simeq 10^{27}$. We shall support this interpretation at the end of this Appendix by analysing the frequency of appearance of such large sized nuggets.

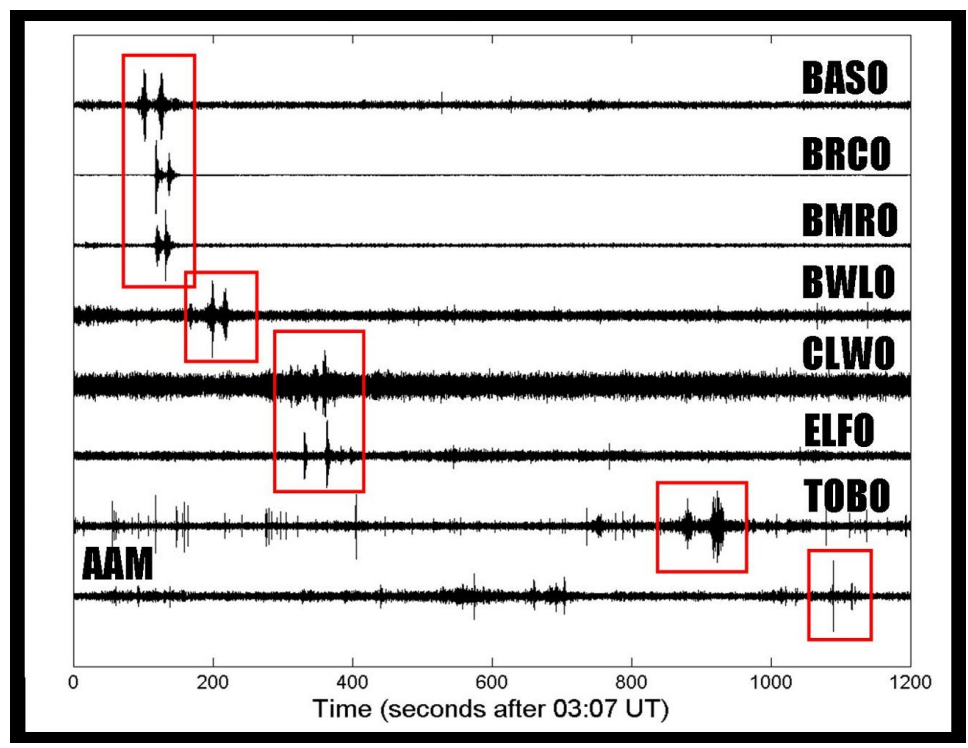

Figure A3. Impulses as observed by seismic stations in the area, adopted from [7], see link in "Data Availability Statement", above the "Acknowledgements".

Another parameter which characterizes the acoustic shock is the frequency determined by the scaling Formula (19). Assuming the same numerical parameters as before we arrive at the following numerical estimate:

$$
v(\bar{x}) \sim \frac{v_{0}}{\bar{x}^{1 / 4}} \sim 5 \mathrm{~Hz}\left(\frac{300 \mathrm{~km}}{r}\right)^{1 / 4},
$$

which is precisely in the range of the highest sensitivity of ELFO where the noise levels are: $10^{-4} \mathrm{~Pa}^{2} / \mathrm{Hz}$ for the $10 \mathrm{~Hz}$ frequency band and $10^{-3} \mathrm{~Pa}^{2} / \mathrm{Hz}$ in the $1 \mathrm{~Hz}$ frequency band [7]. One should emphasize that the accuracy of the estimate (A2) for the frequency is relatively high, about a factor of two or so, as explained after Equation (19). Therefore, a good agreement between estimates (A2) and observations is an encouraging sign for our proposal.

Now our task is to estimate the relevant frequency for the sound emitted in the underground rocks. The corresponding expression for the frequency at $r \simeq 300 \mathrm{~km}$ is determined by the scaling relation (26) and it is given by:

$$
v\left(\bar{x}^{\downarrow}\right) \sim \frac{v_{0}}{\sqrt[4]{\bar{x}^{\downarrow}}} \sim 2.5\left(\frac{1}{\eta}\right)^{\frac{3}{4}} \cdot\left(\frac{300 \mathrm{~km}}{r}\right)^{\frac{1}{4}} \mathrm{kHz} .
$$

The estimate for the underground frequency of Equation (A3) should be contrasted with the estimate of Equation (A2) for the atmosphere. The basic observation is that the acoustic waves in the atmosphere are in the infrasonic frequency range, while underground, they are in the sound frequency band, as already mentioned.

Several comments are in order. First of all, our proposal demonstrates a qualitative consistency with the mysterious event recorded by ELFO on 31 July 2008. Indeed, as we mentioned above the AQNs do not emit the visible light directly, see Appendix B. It should be contrasted with conventional meteors which directly emit the visible light being consistent with black body radiation spectrum. This observation implies that the AQNs 
cannot be observed by conventional optical monitoring. The mysterious event recorded by ELFO on July 31st falls into this category because it was observed by infrasound instruments and not observed by the optical synchronized cameras.

Another qualitative comment goes as follows. The frequency (A3) of the sound from the underground blast falls in human hearing range. Therefore, it is also consistent with the fact that residents of nearby Kincardine could hear the sound (rather than infrasound) signal which originates from the underground with frequency (A3). It is also consistent with seismic observations which are correlated ( The corresponding bangs are classified as seismically coupled "Audible Bangs") with infrasound impulses recorded by ELFO. As discussed above, the atmospheric and underground acoustic waves emitted by the same AQN are always accompanied by each other as they originate from one and the same AQN propagating from outer space through the atmosphere and the Earth.

How we should interpret two blasts recorded in the acoustic and infrasonic frequency bands? The answer depends on the theory of formation of the acoustic waves as a result of ultrasonic motion with very large Mach number which of course is not developed yet. The complicated structure of the signal may, in principle, be explained by the fact that there are actually four waves moving with different speed and originating from different points, which arrive at a different time: atmospheric wave (slow), longitudinal and transverse waves coming from underground, and surface wave. In addition, there are reflected waves (echo). The answering all these hard questions obviously requires an analysis of large number of events, and obviously cannot be accomplished the present time with a single recorded event. However, the basic characteristics such as the frequency and over-pressure for infrasonic signal are consistent with ELFO record.

Furthermore, our estimations are also consistent on the quantitative level with the mysterious event recorded by ELFO on 31 July 2008. Indeed, our estimates for overpressure (A1) and the frequency estimate (A2) are consistent with description [7] represented on Figure A2. This obviously should be considered as a strong support of our identification between a rare intense AQN event and the mysterious explosion recorded by ELFO.

Our final comment is related to the energetics and frequency of appearance of such mysterious events interpreted in terms of the AQNs. As we already mentioned at the end of Section 3 we interpret a relatively high overpressure (A1) at the level of $\delta p \simeq 0.3 \mathrm{~Pa}$ as a result of hitting of a sufficiently large AQN with $B \simeq 10^{27}$ which represents our explanation of why this area has observed a single event in 10 years rather than observing similar events much more often.

\section{Appendix B. AQN Emission Spectrum}

The goal of this Appendix is to overview the spectral characteristics of the AQNs as a result of annihilation events when the nugget enters the Earth atmosphere. The corresponding computations have been carried out in [21] in application to the galactic environment with a typical density of surrounding visible baryons of order $n_{\text {galaxy }} \sim 300 \mathrm{~cm}^{-3}$ in the galactic center. We review these computations with few additional elements which must be implemented for Earth's atmosphere when typical density of surrounding baryons is much higher $n_{\text {air }} \sim 10^{21} \mathrm{~cm}^{-3}$.

The spectrum of nuggets at low temperatures was analyzed in [21] and was found to be,

$$
\begin{array}{r}
\frac{d F}{d \omega}(\omega)=\frac{d E}{d t d A d \omega} \simeq \frac{1}{2} \int_{0}^{\infty} d z \frac{d Q}{d \omega}(\omega, z) \sim \\
\sim \frac{4}{45} \frac{T^{3} \alpha^{5 / 2}}{\pi} \sqrt[4]{\frac{T}{m}}\left(1+\frac{\omega}{T}\right) e^{-\omega / T} h\left(\frac{\omega}{T}\right),
\end{array}
$$

where $\alpha=137^{-1}$ is the fine structure constant and we use units $\hbar=c=k_{B}=1$. The function $Q(\omega, z) \sim n^{2}(z, T)$ describes the emissivity per unit volume from the electrosphere characterized by the density $n(z, T)$, where $z$ measures the distance from the quark core of 
the nugget. The $\frac{d F}{d \omega}(\omega)$ describes the intensity of emission from unit area $A$ at frequency $\omega$ at temperature T. In Equation (A4) a complicated function $h(x)$ can be well approximated as

$$
h(x)= \begin{cases}17-12 \ln (x / 2) & x<1, \\ 17+12 \ln (2) & x \geq 1 .\end{cases}
$$

Integrating over $\omega$ contributes a factor of $T \int d x(1+x) \exp (-x) h(x) \approx 60 T$, giving the total surface emissivity:

$$
F_{\text {tot }}=\frac{d E}{d t d A}=\int_{0}^{\infty} d \omega \frac{d F}{d \omega}(\omega) \sim \frac{16}{3} \frac{T^{4} \alpha^{5 / 2}}{\pi} \sqrt[4]{\frac{T}{m}} .
$$

Although a discussion of black-body radiation is inappropriate for these nuggets (for one thing, they are too small to establish thermal equilibrium with low-energy photons), it is still instructive to compare the form of this surface emissivity with that of black-body radiation $F_{B B}=\sigma T^{4}$

$$
\frac{F_{\mathrm{tot}}}{F_{B B}} \simeq \frac{320}{\pi^{3}} \alpha^{5 / 2} \sqrt[4]{\frac{T}{m}} .
$$

At $T=1 \mathrm{eV}$ which was an appropriate internal nugget's temperature for the galactic environment, the emissivity $F_{\text {tot }} \sim 10^{-6} F_{B B}$ is much smaller than that for black-body radiation. As we discuss below a typical internal nugget's temperature when AQN enters the atmosphere is of order of $T=10 \mathrm{keV}$ which results in the emissivity $F_{\mathrm{tot}} \sim 10^{-5} F_{B B}$ for such high temperatures.

A typical internal temperature of the nuggets can be estimated from the condition the radiative output of Equation (A7) must balanced the flux of energy onto the nugget due to the annihilation events. In this case we may write,

$$
\left(4 \pi R^{2}\right) \frac{16}{3} \frac{T^{4} \alpha^{5 / 2}}{\pi} \sqrt[4]{\frac{T}{m}} \simeq \kappa\left(\pi R^{2}\right) 2 \mathrm{GeV} n_{\mathrm{air}} v_{\mathrm{AQN}},
$$

where the left hand side accounts for the total energy radiation from the nuggets' surface per unit time, while the right hand side accounts for the rate of annihilation events when each successful annihilation event of a single baryon charge produces $\sim 2 m_{p} c^{2} \simeq 2 \mathrm{GeV}$ energy. In Equation (A9) we assume that the nugget is characterized by the geometrical cross section $\pi R^{2}$ when it propagates in environment with local density $n_{\text {air }}$ with velocity $v_{\mathrm{AQN}} \sim 10^{-3} \mathrm{c}$

The factor $\kappa$ is introduced to account for complicated physics as mentioned in the main body of the text. In a neutral environment when no long range interactions exist the value of $\kappa$ cannot exceed $\kappa \sim 1$ which would correspond to the total annihilation of all impacting matter into to thermal photons. The high probability of reflection at the sharp quark matter surface lowers the value of $\kappa$. The propagation of an ionized (negatively charged) nugget in a highly ionized plasma will increase the effective cross section, and therefore value of $\kappa$ as discussed in [11] in application to the solar corona heating problem.

For the neutral environment (such as Earth's atmosphere) and relatively low temperature when the most positrons from electrosphere remain in the system, the parameter $\kappa$ should assume values close to unity, i.e., $0.1 \lesssim \kappa \lesssim 1$. In this case, from (A9) assuming that $0.1 \lesssim \kappa \lesssim 1$ one can estimate a typical internal nugget's temperature in the Earth atmosphere:

$$
T \sim 20 \mathrm{keV} \cdot\left(\frac{n_{\text {air }}}{10^{21} \mathrm{~cm}^{-3}}\right)^{\frac{4}{17}}\left(\frac{\kappa}{0.1}\right)^{\frac{4}{17}}
$$

where we use typical air density $n_{\text {air }}$ and $v_{\mathrm{AQN}} \sim 10^{-3} \mathrm{c}$. The internal temperature $T$ in this simple setting does not depend on the size of the nugget $R$ as emission and collision rates are both proportional to $R^{2}$. Thus, in the air the temperature lies in the range 
$T \simeq(20-40) \mathrm{KeV}$, depending on parameter $\kappa$. In case of solids the temperature must be higher because of higher density. This leads to a stronger AQN-ionization. This attracts more positively charged ions from surrounding material, which consequently may increase the rate of annihilation (effectively increasing $\kappa$ ). All these effects are very complicated at large $T$ and deserve a separate study. In the present work for the order of magnitude estimates we adopt the previous value of $0.1 \lesssim \kappa \lesssim 1$ for the case of solids as well, which would correspond to $T \simeq(100-200) \mathrm{KeV}$. All the uncertainties related to $\kappa$ do not modify our qualitative order of magnitude estimates of this work.

There are few additional elements which should be taken into account for Earth's atmosphere in comparison with original computations [21,22] applied to very dilute galactic environment with much lower temperatures $T \simeq 1 \mathrm{eV}$. These effects do not modify the basic scale (A10). However, these additional elements strongly affect the spectrum at the lower frequency bands. In particular the visible portion of the spectrum at $\omega \sim 1 \mathrm{eV}$ demonstrates a dramatic suppression. It has some profound consequences for the present work as discussed in the main body of the text. In particular, it implies that the AQNs do not emit the visible light with $\omega \sim 1 \mathrm{eV}$, in huge contrast with conventional meteors and meteorites which are normally characterized by strong emission in the visible frequency bands through sputtering and ablation $[13,18,19]$.

We start our analysis on additional elements to be implemented with the plasma frequency $\omega_{p}$ which characterizes the propagation of photons in the ionized plasma, which represents the electrosphere for our AQN system. The $\omega_{p}$ can be thought as an effective mass for photon: only photons with the energy larger than this mass can propagate outside of the system, while photons with $\omega<\omega_{p}$ can only propagate for a short time/distance $\sim \omega_{p}^{-1}$ before they get absorbed by the plasma. For our estimates we shall use a conventional non-relativistic expression for $\omega_{p}$ :

$$
\omega_{p}^{2}(z, T)=\frac{4 \pi \alpha n(z, T)}{m},
$$

where $m$ is the electron mass. The positron density $n(z, T)$ in electrosphere in the nonrelativistic mean-field approximation has been computed in [21,22]:

$$
n(z, T) \simeq \frac{T}{2 \pi \alpha} \cdot \frac{1}{(z+\bar{z})^{2}}, \frac{1}{\bar{z}} \simeq \sqrt{2 \pi \alpha} m \sqrt[4]{\frac{T}{m}},
$$

where $\bar{z}$ is a constant of integration being determined by appropriate boundary condition deep inside the nugget's core. Important implication of the plasma frequency $\omega_{p}(z, T)$ is that the very dense regions in electrosphere essentially do not emit the photons with $\omega \lesssim \omega_{p}(z, T)$.

There is another effect which further suppresses the emission of low energy photons. It is related to the ionization processes when the AQN assumes a sufficiently large negative charge due to the $T \neq 0$ as estimated above (A10). Essentially it affects all loosely bound positrons which will be completely stripped off by high temperature, while more strongly bound positrons will be less affected by the same temperature. The corresponding effect leads to very strong suppression of low energy photons with $\omega \ll T$ as loosely bound positrons represent the main source of low frequency photons.

Both these effects have been implemented in Equation (A4) by performing numerical computation of the integral $\int d z$ over electrosphere region with corresponding modifications of the positron density $n(z, T)$ and inserting $\omega_{p}(z, T)$ as discussed above. We present the corresponding results for these numerical studies on Figure A4 for $T=10 \mathrm{keV}$ and $T=50 \mathrm{keV}$. These values for the temperature essentially cover the most relevant window (A10) for the present analysis which dealt with AQN emission in atmosphere.

Few comments are in order. First of all, as one can see from Figure A4 the spectrum is almost flat in the region $\omega \lesssim T$ which is the direct manifestation of the well known soft photon theorem when the emission of the photon with frequency $\omega$ is proportional to $d \omega / \omega$. 
For large $\omega \gg T$ the exponential suppression $\exp (-\omega / T)$ becomes the most important element of the spectrum. The emission is strongly suppressed at very small $\omega \simeq \omega_{p} \ll T$. The strong suppression of the spectrum with $\omega \ll T$ has profound phenomenological consequences: drastic intensity drop at small $\omega \ll T$ implies that that the luminosity of the visible light from $\mathrm{AQN}$ with $\omega \sim(1-10) \mathrm{eV}$ is strongly suppressed in comparison with $X$-ray emission. This strong suppression is entirely due to the two effects mentioned above: the presence of the plasma frequency in electrosphere (A11) and complete stripping off of the loosely bound positrons. It implies that the AQNs cannot be observed by conventional optical monitoring as AQNs are not accompanied by emission of the visible light. It should be contrasted with meteors and meteorites which are normally characterized by strong emission in the visible frequency bands through sputtering and ablation $[13,18,19]$ and routinely observed by all-sky cameras.

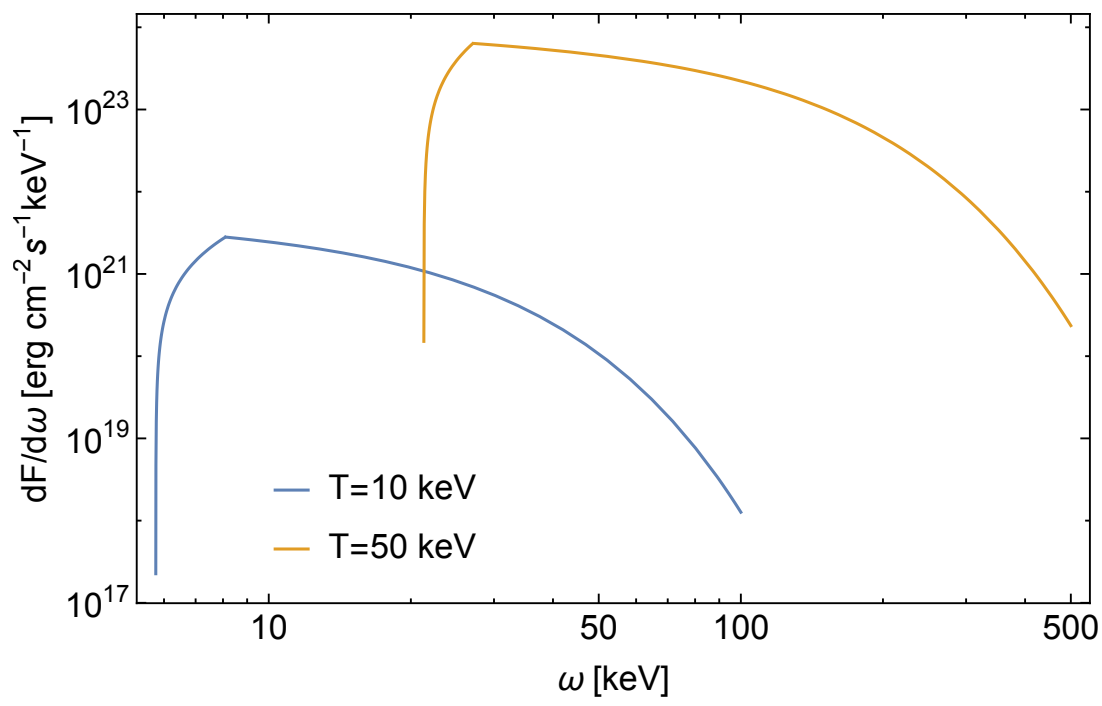

Figure A4. The spectral surface emissivity of a nugget with the suppression effects at $\omega \ll T$ as discussed in this Appendix. The blue solid line corresponds to $T=10 \mathrm{keV}$ while the yellow line corresponds to $T=50 \mathrm{keV}$. The internal temperature $T$ is determined by the density of the atmosphere and parameter $\kappa$ according to (A10).

\section{References}

1. Zhitnitsky, A.R. 'Nonbaryonic' dark matter as baryonic colour superconductor. J. Cosmol. Astropart. Phys. 2003, 10, 10. [CrossRef]

2. Witten, E. Cosmic separation of phases. Phys. Rev. D 1984, 30, 272-285. [CrossRef]

3. Farhi, E.; Jaffe, R.L. Strange matter. Phys. Rev. D 1984, 30, 2379-2390. [CrossRef]

4. De Rujula, A.; Glashow, S.L. Nuclearites-A novel form of cosmic radiation. Nature 1984, 312, 734-737. [CrossRef]

5. Madsen, J. Physics and Astrophysics of Strange Quark Matter. In Hadrons in Dense Matter and Hadrosynthesis; Cleymans, J., Geyer, H.B., Scholtz, F.G., Eds.; Lecture Notes in Physics; Springer: Berlin, Germany, 1999; Volume 516, p. 162. [CrossRef]

6. Zhitnitsky, A. Axion quark nuggets. Dark matter and matter-antimatter asymmetry: Theory, observations and future experiments. Mod. Phys. Lett. A 2021, 36, 2130017. [CrossRef]

7. The Elginfield Infrasound Array (ELFO). Meteor Infrasound. Available online: https://aquarid.physics.uwo.ca/research/ infrasound/is_mysteriousexplosions.html (accessed on 11 January 2022).

8. Lawson, K.; Liang, X.; Mead, A.; Siddiqui, M.S.R.; Van Waerbeke, L.; Zhitnitsky, A. Gravitationally trapped axions on the Earth. Phys. Rev. D 2019, 100, 043531. [CrossRef]

9. Herrin, E.T.; Rosenbaum, D.C.; Teplitz, V.L. Seismic search for strange quark nuggets. Phys. Rev. D 2006, 73, 043511. [CrossRef]

10. Zhitnitsky, A. Solar Extreme UV radiation and quark nugget dark matter model. J. Cosmol. Astropart. Phys. 2017, 10, 050. [CrossRef]

11. Raza, N.; van Waerbeke, L.; Zhitnitsky, A. Solar corona heating by axion quark nugget dark matter. Phys. Rev. D 2018, $98,103527$. [CrossRef]

12. Ge, S.; Lawson, K.; Zhitnitsky, A. Axion quark nugget dark matter model: Size distribution and survival pattern. Phys. Rev. D 2019, 99, 116017. [CrossRef]

13. Silber, E.A.; Boslough, M.; Hocking, W.K.; Gritsevich, M.; Whitaker, R.W. Physics of meteor generated shock waves in the Earth's atmosphere-A review. Adv. Space Res. 2018, 62, 489-532. [CrossRef] 
14. Zhitnitsky, A. Cold dark matter as compact composite objects. Phys. Rev. D 2006, 74, 043515. [CrossRef]

15. Flambaum, V.V.; Zhitnitsky, A.R. Primordial Lithium Puzzle and the Axion Quark Nugget Dark Matter Model. Phys. Rev. D 2019, 99, 023517. [CrossRef]

16. Lawson, K.; Zhitnitsky, A.R. The $21 \mathrm{~cm}$ absorption line and the axion quark nugget dark matter model. Phys. Dark Univ. 2019, 24, 100295. [CrossRef]

17. ReVelle, D. on meteor-generated infrasound. J. Geophys. Res. Planets 1976, 81, 1217. [CrossRef]

18. Silber, E.A.; Brown, P.G. Optical observations of meteors generating infrasound-I: Acoustic signal identification and phenomenology. J. Atmos. Sol. Terr. Phys. 2014, 119, 116-128. [CrossRef]

19. Silber, E.A.; Brown, P.G.; Krzeminski, Z. Optical observations of meteors generating infrasound: Weak shock theory and validation. J. Geophys. Res. Planets 2015, 120, 413-428. [CrossRef]

20. Tanabashi, M. Review of Particle Physics. Phys. Rev. D 2018, 98, 030001. [CrossRef]

21. Forbes, M.M.; Zhitnitsky, A.R. WMAP Haze: Directly Observing Dark Matter? Phys. Rev. D 2008, 78, 083505. [CrossRef]

22. Forbes, M.M.; Lawson, K.; Zhitnitsky, A.R. The Electrosphere of Macroscopic 'Quark Nuclei': A Source for Diffuse MeV Emissions from Dark Matter. Phys. Rev. D 2010, 82, 083510. [CrossRef]

23. FjASr, E.; Holt, R.; Horsrud, P.; Raaen, A.; Risnes, R. Chapter 5 Elastic wave propagation in rocks. In Petroleum Related Rock Mechanics, 2nd ed.; FjASr, E., Holt, R., Horsrud, P., Raaen, A., Risnes, R., Eds.; Elsevier: Amsterdam, The Netherlands, 2008; Volume 53, pp. 175-218. [CrossRef]

24. Landau, L.D.; Lifshitz, E.M. Fluid Mechanics, 2nd ed.; Butterworth-Heinemann: Oxford, UK, 1987; Volume 6. [CrossRef]

25. Absorption Length Calculator. Available online: http:/ / resource.npl.co.uk/acoustics/techguides/seaabsorption/ (accessed on 11 January 2022).

26. Parker, T.; Shatalin, S.; Farhadiroushan, M. Distributed Acoustic Sensing-A new tool for seismic applications. First Break 2014, 32, 61. [CrossRef]

27. Daley, T.; Miller, D.E.; Dodds, K.; Cook, P.; Freifeld, B.M. Field testing of modular borehole monitoring with simultaneous distributed acoustic sensing and geophone vertical seismic profiles at Citronelle, Alabama. Geophys. Prospect. 2016, 64, 1318. [CrossRef]

28. Jousset, P.; Reinsch, T.; Ryberg, T.; Blanck, H.; Clarke, A.; Aghayev, R.; Hersir, G.P.; Henninges, J.; Weber, M.; Krawczyk, C.M. Dynamic strain determination using fibre-optic cables allows imaging of seismological and structural features. Nat. Commun. 2018, 9, 2509. [CrossRef] [PubMed]

29. Ajo-Franklin, J.B.; Dou, S.; Lindsey, N.J.; Monga, I.; Tracy, C.; Robertson, M.; Rodriguez Tribaldos, V.; Ulrich, C.; Freifeld, B.; Daley, T.; et al. Distributed Acoustic Sensing Using Dark Fiber for Near-Surface Characterization and Broadband Seismic Event Detection. Sci. Rep. 2019, 9, 1328. [CrossRef] [PubMed]

30. Budker, D.; Flambaum, V.V.; Liang, X.; Zhitnitsky, A. Axion Quark Nuggets and how a Global Network can discover them. Phys. Rev. D 2020, 101, 043012. [CrossRef]

31. Freese, K.; Frieman, J.; Gould, A. Signal modulation in cold-dark-matter detection. Phys. Rev. D 1988, 37, 3388-3405. [CrossRef] [PubMed]

32. Freese, K.; Lisanti, M.; Savage, C. Colloquium: Annual modulation of dark matter. Rev. Mod. Phys. 2013, 85, 1561-1581. [CrossRef]

33. Liang, X.; Mead, A.; Siddiqui, M.S.R.; Van Waerbeke, L.; Zhitnitsky, A. Axion Quark Nugget Dark Matter: Time Modulations and Amplifications. Phys. Rev. D 2020, 101, 043512. [CrossRef]

34. Liang, X.; Peshkov, E.; Van Waerbeke, L.; Zhitnitsky, A. Proposed network to detect axion quark nugget dark matter. Phys. Rev. D 2021, 103, 096001. [CrossRef]

35. Incorporated Research Institutions for Seismology (IRIS) Network. Available online: https://www.iris.edu/hq/programs/gsn (accessed on 11 January 2022).

36. Zhitnitsky, A. The Mysterious Bursts observed by Telescope Array and Axion Quark Nuggets. J. Phys. G Nucl. Part Phys. 2020. [CrossRef]

37. Zhitnitsky, A. Multi-Modal Clustering Events Observed by Horizon-10T and Axion Quark Nuggets. Universe 2021, 7, 384. [CrossRef] 